\title{
Human Platelet Antigen Alleles in 998 Taiwanese Blood Donors Determined by Sequence-Specific Primer Polymerase Chain Reaction
}

\author{
Shun-Chung Pai, ${ }^{1,2}$ Thierry Burnouf, ${ }^{3,4}$ Jen-Wei Chen, ${ }^{2}$ and Liang-In Lin ${ }^{1,5}$ \\ ${ }^{1}$ Department of Clinical Laboratory Sciences and Medical Biotechnology, College of Medicine, National Taiwan University, \\ No. 1 Chang-Te Street, Taipei 10048, Taiwan \\ ${ }^{2}$ Taiwan Blood Services Foundation, 3rd Floor No. 3 Nan-Hai Road, Taipei 10066, Taiwan \\ ${ }^{3}$ Institute of Biomedical Materials and Tissue Engineering, College of Oral Medicine, Taipei Medical University, Taipei 11031, Taiwan \\ ${ }^{4}$ Human Protein Process Sciences (HPPS), 59000 Lille, France \\ ${ }^{5}$ Department of Laboratory Medicine, National Taiwan University Hospital, No. 7 Chung-Shan Southern Road, Taipei 10016, Taiwan
}

Correspondence should be addressed to Liang-In Lin; lilin@ntu.edu.tw

Received 8 April 2013; Accepted 10 June 2013

Academic Editor: Jeffrey A. Frelinger

\begin{abstract}
Copyright $\odot 2013$ Shun-Chung Pai et al. This is an open access article distributed under the Creative Commons Attribution License, which permits unrestricted use, distribution, and reproduction in any medium, provided the original work is properly cited.

Polymorphism of human platelet antigens (HPAs) leads to alloimmunizations and immune-mediated platelet disorders including fetal-neonatal alloimmune thrombocytopenia (FNAIT), posttransfusion purpura (PTP), and platelet transfusion refractoriness (PTR). HPA typing and knowledge of antigen frequency in a population are important in particular for the provision of HPAmatched blood components for patients with PTR. We have performed allele genotyping for HPA-1 through - 6 and -15 among 998 platelet donors from 6 blood centers in Taiwan using sequence-specific primer polymerase chain reaction. The HPA allele frequency was 99.55 , and $0.45 \%$ for HPA-la and $-1 \mathrm{~b}$; 96.49 , and $3.51 \%$ for HPA-2a and -2b; 55.81, and 44.19\% for HPA-3a and 3b; 99.75, and $0.25 \%$ for HPA-4a and $-4 \mathrm{~b}$; 98.50 , and $1.50 \%$ for HPA-5a and $-5 \mathrm{~b}$; 97.75 and 2.25\% for HPA-6a and $-6 \mathrm{~b}$; 53.71 and 46.29\% for HPA-15a and -15b. HPA-15b and HPA-3a, may be considered the most important, followed by HPA-2, $-6,-1,-5$, and -4 systems, as a cause of FNAIT, PTP, and PTR based on allele frequency. HPA- $4 \mathrm{~b}$ and HPA-5b role cannot be excluded based on their immunogenicity. A larger-scale study will now be conducted to confirm these hypotheses and to establish an apheresis donor database for the procurement of HPA-matched apheresis platelets for patients with PTR.
\end{abstract}

\section{Introduction}

Human platelet antigens (HPAs) are polymorphic structures located on platelet membrane glycoproteins. As is the case for $\mathrm{ABO}$ and $\mathrm{Rh}$ blood groups, platelet antigen frequency varies among ethnic groups. HPA polymorphism leads to the occurrence of human platelet antibodies following alloimmunization against HPA [1]. HPA alloimmunization is responsible for clinically relevant immune-mediated platelet disorders including fetal/neonatal alloimmune thrombocytopenia (FNAIT), posttransfusion purpura (PTP), and platelet transfusion refractoriness (PTR) [2-4]. HPA genotyping in different populations is of importance for predicting the risk of HPA alloimmunization in different ethnic groups [5].
This is essential, as a complement to clinical history, for FNAIT diagnosis and possible future prevention [6-8] and for provision of HPA-matched blood components for patients with PTR $[4,9]$.

The most extensive studies on HPA gene frequencies have so far been carried out in North America and Western Europe. They have established that, in Caucasians, HPAla, located on integrin- $\beta 3$, is responsible for around $85 \%$ of FNAIT cases caused by HPA antibodies [10]. However, estimates of HPA frequency in other populations in the world are still incomplete. In particular, data among oriental populations, including Taiwanese, are still insufficient. In this study, we used sequence-specific primers polymerase chain reaction (SSP-PCR) to screen 998 Taiwanese platelet 
apheresis donors for 7 clinically significant biallelic human platelet antigens, HPA-1 through -6 and -15 . Our goal was (a) to understand which HPA may likely cause FNAIT and (b) to obtain blood donors data to be able to provide HPAmatched platelets for patients with PTR in Taiwan. Since these transfusions may often be required on short notice, it is indeed important to have previously identified suitable donors.

\section{Material and Methods}

2.1. Blood Samples and DNA Extraction. 998 randomly selected healthy volunteer apheresis platelet donors of six blood centers of the Taiwan Blood Services Foundation (TBSF; Taipei: 325 donors; Hsinchu: 101; Taichung: 97; Tainan: 93; Kaohsiung: 332; and Hualien: 50) were recruited in the study. All the subjects gave informed consent. Blood samples of $3 \mathrm{~mL}$ were collected in ethylenediaminetetraacetic acid (EDTA) anticoagulant vacuum tubes and frozen in polypropylene vials at $-70^{\circ} \mathrm{C}$. DNA was extracted from $0.2 \mathrm{~mL}$ of EDTA-treated blood using commercial DNA extraction kit (Texas BioGene Inc., Richardson, TX, USA) and processed according to the manufacturer's instructions. The eluted DNA was diluted to a concentration of $15 \mathrm{ng} / \mu \mathrm{L}$.

\subsection{Sequence-Specific Primer Polymerase Chain Reaction} (SSP-PCR). HPA typing was conducted using SSP-PCR. Samples were tested with the commercial Platelet SSP HPA1 to -6 and -15 Typing Kit (Texas BioGene Inc., Richardson, TX, USA) according to the manufacturer's instructions.

2.3. Gel Electrophoresis and Gel Interpretation. Amplified DNA products were visualized by UV illumination followed by electrophoresis on $2.0 \%$ agarose gel. Results were recorded by ultraviolet photography. For each polymorphic position, one or two possible patterns might be observed: $a$ or $b$ genotype. Samples were classified as HPA a/a, a/b, or b/b genotypes.

2.4. HPA Genotyping by Sequence-Based Typing Method (SBT). To verify the SSP-PCR results, 20 DNA samples were selected for genotyping for HPA- 1 to -6 and -15 by SBT. Sequencing primers were designed according to previously published sDNA sequences [11]. DNA samples were amplified, followed by bidirectional sequencing of the amplicons using the ABI BigDye Terminator Cycle Sequencing kit (Applied Biosystems, Foster City, CA, USA) according to the manufacturer's instructions. The sequencing reaction products were electrophoresed using an ABI Prism sequencer (Applied Biosystems). The sequence data were analyzed by SeqScape 2.5 software (Applied Biosystems).

2.5. Statistical Analysis. HPA genotype frequencies were estimated by direct counting, and allele frequencies and heterozygosity were calculated by the genetic marker data analysis software (PowerMarker) [12]. Tests for Hardy-Weinberg equilibrium in the population were performed by $\chi^{2}$ test. Mismatch probability of different platelet alloantigens, after random platelet concentrate transfusions, was calculated [13] by using the formula $2 a b(1-a b)$, where $a$ and $b$ are the respective HPA gene frequencies at a diallelic focus. The probability of fetomaternal incompatibility between pregnant females and their fetuses regarding different HPA was calculated [14] for assessing the probability of HPA alloantibody development. Comparison between the gene frequencies of six blood centers was done with $\chi^{2}$ test using SPSS version 14 (SPSS Inc., Chicago, IL, USA). Where the number of any variable was $<5$, Fischer's exact test was used to determine the $P$ value between two groups.

\section{Results}

3.1. HPA Genotyping by SBT. The genotyping results obtained by SSP-PCR were validated using genotyping data by SBT. No discrepancies were observed between the SSP-PCR and SBT typing for HPA-1 to -6 and -15 systems in 20 selected DNA samples, indicating the reliability of the SSP-PCR results.

\subsection{The Genotype Distributions and Allele Frequency for} HPA-1 to -6 and -15. The genotype and allele frequency of HPAs from the 998 apheresis platelet donors are shown in Table 1. HPA-3 and -15 systems exhibited the greatest heterozygosity among all HPA systems. The frequency of $\mathrm{a} / \mathrm{a}, \mathrm{a} / \mathrm{b}$, and $\mathrm{b} / \mathrm{b}$ genotypes was $29.7 \%, 52.3 \%$, and $18.0 \%$ in the HPA-3 system and $28.4 \%, 50.7 \%, 20.9 \%$ in the HPA15 system, respectively. The frequency of HPA-1a/a (99.1\%), 2a/a (93.2\%), 4a/a (99.5\%), 5a/a (97.0\%), and 6a/a (95.6\%) homozygous genotypes was higher than those of HPA-1a/b $(0.9 \%), 2 \mathrm{a} / \mathrm{b}(6.6 \%), 4 \mathrm{a} / \mathrm{b}(0.5 \%), 5 \mathrm{a} / \mathrm{b}(3.0 \%)$, and $6 \mathrm{a} / \mathrm{b}(4.3 \%)$ heterozygous genotypes. Only two individuals with HPA$2 \mathrm{~b} / \mathrm{b}$ and one with HPA-6b/b had a "rare" genotype, and no $\mathrm{b} / \mathrm{b}$ homozygote was found in the HPA-1, -4 , and -5 systems. There was no significant deviation from the Hardy-Weinberg equilibrium in any of the systems studied in this population. Moreover, the "a" allele was dominant, and the "b" allele was infrequent, in these HPA systems, except for HPA-3 and HPA15 , with frequencies of $55.8 \%, 44.2 \%$ for HPA-3a, HPA-3b and $53.7 \%, 46.3 \%$ for HPA-15a, HPA-15b, respectively. Allele frequency did not differ among blood centers, possibly apart from HPA-6 (not shown).

3.3. Estimated Probability of Incompatibility Leading to Risks of FNAIT and PTR. The probability of alloimmunization against the HPA 1 to -6 and -15 systems was estimated as a way to assess the risk of FNAIT and PTR occurrence in Taiwan (Table 2). The probability of fetomaternal mismatch in HPA-1, -2, -4, -5, and -6 was very low. In HPA-3a/a and $-3 \mathrm{~b} / \mathrm{b}$ mothers, the risk of mismatch with the fetal antigens during pregnancy was found to be 138/1000 and $109 / 1000$, respectively. It was $133 / 1000$ pregnancies for an HPA-15a/a mother, and 115/1000 pregnancies for an HPA$15 \mathrm{~b} / \mathrm{b}$ mother. The mismatch probability of HPA after random platelet transfusions ranged from 0 to 0.37 . The highest risks of incompatibility, in HPA-3 and - 15 systems, were 37.16 and $37.76 \%$, respectively, whereas the chances of incompatibility to HPA-1, $-2,-4,-5$, and -6 were much lower $(<7 \%)$. These 
TABLE 1: Genotype and allele frequency of HPA in Taiwanese $(n=998)$.

\begin{tabular}{lccccccc}
\hline \multirow{2}{*}{ HPA system } & \multicolumn{3}{c}{ Genotype } & \multicolumn{3}{c}{ Allele } & \multicolumn{3}{c}{ Hardy-Weinberg equilibrium } \\
& $\mathrm{a} / \mathrm{a}(\%)$ & $\mathrm{a} / \mathrm{b}(\%)$ & $\mathrm{b} / \mathrm{b}(\%)$ & $\mathrm{a}(\%)$ & $\mathrm{b}(\%)$ & $\chi^{2}$ & 0.0205 \\
HPA-1 & $989(99.10)$ & $9(0.90)$ & $0(0.00)$ & $1987(99.55)$ & $9(0.45)$ & 0.9898 \\
HPA-2 & $930(93.18)$ & $66(6.61)$ & $2(0.20)$ & $1926(96.49)$ & $70(3.51)$ & 0.5222 & 0.7702 \\
HPA-3 & $296(29.65)$ & $522(52.30)$ & $180(18.04)$ & $1114(55.81)$ & $882(44.19)$ & 3.6431 & 0.1618 \\
HPA-4 & $993(99.50)$ & $5(0.50)$ & $0(0.00)$ & $1991(99.75)$ & $5(0.25)$ & 0.0063 & 0.9969 \\
HPA-5 & $968(96.99)$ & $30(3.01)$ & $0(0.00)$ & $1966(98.50)$ & $30(1.50)$ & 0.2324 & 0.8903 \\
HPA-6 & $954(95.59)$ & $43(4.31)$ & $1(0.10)$ & $1951(97.75)$ & $45(2.25)$ & 0.5010 & 0.7784 \\
HPA-15 & $283(28.36)$ & $506(50.70)$ & $209(20.94)$ & $1072(53.71)$ & $924(46.29)$ & 0.3847 & 0.8250 \\
\hline
\end{tabular}

TABLE 2: Estimate of HPA incompatibility probability for assessing the risk of FNAIT and PTR.

\begin{tabular}{lccccc}
\hline & \multirow{2}{*}{ Frequency major allele (\%) } & Heterozygosity (\%) & \multicolumn{2}{c}{ Fetomaternal incompatibility } & Mismatch platelet transfusion \\
& & & Anti-a risk (\%) & Anti-b risk (\%) & 0.89 \\
HPA-1 & 99.55 & 0.90 & $<0.01$ & 0.45 & 6.54 \\
HPA-2 & 96.49 & 6.61 & 0.12 & 3.27 & 37.16 \\
HPA-3 & 55.81 & 52.30 & 10.90 & 13.76 & 0.50 \\
HPA-4 & 99.75 & 0.50 & 0.01 & 0.25 & 2.92 \\
HPA-5 & 98.50 & 2.96 & 0.05 & 2.15 & 4.31 \\
HPA-6 & 97.75 & 4.41 & 11.51 & 13.35 & 37.76 \\
HPA-15 & 53.71 & 49.73 & & & \\
\hline
\end{tabular}

results may help predict the risk of alloimmunization in the patients who will receive random platelet transfusion.

3.4. Allele Frequency of Antigens on GPIIIa. GPIIIa is the most polymorphic glycoproteins on human platelet membranes and it bears in particular HPA-1, HPA-4, and HPA-6. Alloantigenic epitopes can induce an alloimmune response. The gene frequency of the allelic forms of these three antigens in Taiwanese is shown in Table 3.

\section{Discussion}

Platelet-specific antibodies may be involved in alloimmune disorders such as FNAIT, PTP, and PTR [15]. HPA genotyping is important for the diagnosis of alloimmune thrombocytopenic syndromes [16]. The distribution frequency of HPA varies among populations worldwide. Data on HPA frequency in Taiwan population have so far been limited and involved small cohorts. We therefore determined the gene frequency of HPA to be close to one thousand apheresis platelet donors from six blood centers located all over the country. To carry out this study, we used SSP-PCR, the recommended method that has proved to be a fast and efficient way to genotype human leukocyte antigen (HLA) and some HPAs [17]. Commercial kits for HPA genotyping have been made available. Here, we used HPA type Platelet SSP HPA-1 to -6 and -15 Typing Kit for HPA genotyping of these 7 HPAs.

In Caucasian population, HPA-1 is known to be the most important antigen system involved in antiplatelet alloimmunity [15]. In this study, no HPA-1b/1b homozygote was found, which is consistent with previous studies in Taiwan
TABLE 3: Allele frequencies of three platelet antigens located on GPIIIa in Taiwanese.

\begin{tabular}{lcccc}
\hline HPA-1 & HPA-4 & HPA-6 & $\begin{array}{c}\text { Allelic } \\
\text { forms }\end{array}$ & Frequencies \\
\hline Leu33 & Arg143 & Arg489 & 1a/4a/6a & 0.9704 \\
Leu33 & Arg143 & Gln489 & $1 \mathrm{a} / 4 \mathrm{a} / 6 \mathrm{~b}$ & 0.0225 \\
Pro33 & Arg143 & Arg489 & $1 \mathrm{~b} / 4 \mathrm{a} / 6 \mathrm{a}$ & 0.0045 \\
Leu33 & Gln143 & Arg489 & $1 \mathrm{a} / 4 \mathrm{~b} / 6 \mathrm{a}$ & 0.0025 \\
\hline
\end{tabular}

groups $[18,19]$. Only nine individuals were found to be HPA-1a/1b heterozygous. These data likely explain the low incidence of FNAIT and PTP due to HPA-1 alloimmunization in Taiwanese. We identified two homozygous b/b genotype individuals for HPA-2, a new observation for Taiwan [18, 19]. Although there is no report of HPA-2 involvement in alloimmune disorders in Taiwan, it may possibly play a more important role than does HPA-1. The genotype frequency of HPA-3a and -3b confirms previous data [18, 19], but immunization against HPA-3a may be more problematic as it is more immunogenic than HPA-3b. No homozygous $\mathrm{HPA}-4 \mathrm{~b} / \mathrm{b}$ was found in the present study whereas only five heterozygous HPA-4a/b individuals were identified. HPA-5 was considered the second most important immunogenic factor linked with alloimmune syndromes in Caucasians [20]. But in this study, as before [18], no HPA-5b/b was found. The rarity of homozygous HPA-5b/b and the low frequency of HPA-5b may contribute to reducing the probability of platelet incompatibility phenomena involving this antigen in Taiwanese. We found 1 in 998 individuals having HPA-6b/b in 
the present study while none could be found in 1000 individuals from China [13]. A larger-scale study would be needed to identify whether a difference of HPA-6b/b genotype does exist between Taiwanese and the other Chinese groups. HPA15, also known as Gov a/b, is considered to be the most relevant HPA after HPA-1 for FNAIT induction [21], especially HPA-15b that is more immunogenic [20]. A report of 605 samples has shown that the Gov a/b platelet alloantigens have an immunogenicity similar to that of HPA-5 alloantigens [22]. In this study, the genotype frequencies of HPA-15a and $-15 \mathrm{~b}$ are 53.71 and $46.29 \%$, respectively, equivalent to that reported by the Chinese study [13]. The frequency of HPA$1 \mathrm{a} / 4 \mathrm{a} / 6 \mathrm{~b}$ on GPIIIa glycoprotein was about 0.023 , which is higher than that $(0.013)$ reported in Chinese Han population [23] and similar to that (0.027) in Japanese [24]. It should be kept in mind that allele frequency is only one of the factors involved in the risks of platelet antigen alloimmunization [25]. Indeed, while the likelihood of antigen incompatibility between mother and fetus is high in HPA-3 and HPA-15 systems, considering the high heterozygosity rate, they may actually be less likely to cause FNAIT compared to other systems. We found that $0.5 \%$ of individuals are HPA- $4 \mathrm{~b}$ positive (compared to $0 \%$ in Caucasians) and 3.01\% are HPA$5 \mathrm{~b}$ positive; the potential for HPA- $4 \mathrm{~b}$ and HPA-5b to induce alloimmunization cannot be ignored since these alleles are known to be immunogenic $[20,25]$. They may therefore be responsible for more FNAIT cases than HPA-3a or HPA$15 b$.

In conclusion, our data provide information on platelet antigen allele frequency in Taiwan population that can serve as a background (a) to diagnose the cause of FNAIT, PTP, and PTR and (b) to provide HPA-matched apheresis platelet for patients with HPA alloimmune disorders. Large-scale study will now be conducted to confirm these data in order to establish an apheresis donor database for the provision of HPA-matched platelets for transfusion to patients with alloimmune disorders.

\section{Conflict of Interests}

The authors declare that they have no conflict of interests.

\section{Acknowledgment}

The authors thank the Molecular Typing Laboratory at Medigen Biotechnology Corp. for the technical support.

\section{References}

[1] T. J. Kunicki and P. J. Newman, "The molecular immunology of human platelet proteins,” Blood, vol. 80, no. 6, pp. 1386-1404, 1992.

[2] H. Deckmyn, H. Ulrichts, G. Van De Walle, and K. Vanhoorelbeke, "Platelet antigens and their function," Vox Sanguinis, vol. 87, no. 2, pp. S105-S111, 2004.

[3] H. Kroll, V. Kiefel, and S. Santoso, "Clinical aspects and typing of platelet alloantigens," Vox Sanguinis, vol. 74, no. 2, pp. 345354, 1998.
[4] P. A. Pappalardo, A. R. Secord, P. Quitevis, M. D. Haimowitz, and D. Goldfinger, "Platelet transfusion refractoriness associated with HPA-1a ( $\mathrm{Pl}(\mathrm{Al})$ ) alloantibody without coexistent HLA antibodies successfully treated with antigen-negative platelet transfusions," Transfusion, vol. 41, no. 8, pp. 984-987, 2001.

[5] B. R. Curtis, "Genotyping for human platelet alloantigen polymorphisms: applications in the diagnosis of alloimmune platelet disorders," Seminars in Thrombosis and Hemostasis, vol. 34, no. 6, pp. 539-548, 2008.

[6] H. Tiller, M. K. Killie, P. Chen et al., "Toward a prophylaxis against fetal and neonatal alloimmune thrombocytopenia: induction of antibody-mediated immune suppression and prevention of severe clinical complications in a murine model," Transfusion, vol. 52, no. 7, pp. 1446-1457, 2012.

[7] J. Kjeldsen-Kragh, H. Ni, and B. Skogen, “Towards a prophylactic treatment of HPA-related foetal and neonatal alloimmune thrombocytopenia," Current Opinion in Hematology, vol. 19, no. 6, pp. 469-474, 2012.

[8] B. Skogen, M. K. Killie, J. Kjeldsen-Kragh et al., "Reconsidering fetal and neonatal alloimmune thrombocytopenia with a focus on screening and prevention," Expert Review of Hematology, vol. 3, no. 5, pp. 559-566, 2010.

[9] V. M. J. Novotny, "Prevention and management of platelet transfusion refractoriness," Vox Sanguinis, vol. 76, no. 1, pp. 1-13, 1999.

[10] M. F. Murphy and J. B. Bussel, "Advances in the management of alloimmune thrombocytopenia," British Journal of Haematology, vol. 136, no. 3, pp. 366-378, 2007.

[11] M. Liew, L. Nelson, R. Margraf et al., "Genotyping of human platelet antigens 1 to 6 and 15 by high-resolution amplicon melting and conventional hybridization probes," Journal of Molecular Diagnostics, vol. 8, no. 1, pp. 97-104, 2006.

[12] K. Liu and S. V. Muse, "PowerMaker: an integrated analysis environment for genetic maker analysis," Bioinformatics, vol. 21, no. 9, pp. 2128-2129, 2005.

[13] M. L. Feng, D. Z. Liu, W. Shen et al., "Establishment of an HPA1- to -16-typed platelet donor registry in China," Transfusion Medicine, vol. 16, no. 5, pp. 369-374, 2006.

[14] H. Ohto, T. Yamaguchi, C. Takeuchi, Y. Tohyama, A. Sato, and S. Morita, "Anti-HPA-5b-induced neonatal alloimmune thrombocytopenia: antibody titre as a predictor," British Journal of Haematology, vol. 110, no. 1, pp. 223-227, 2000.

[15] V. M. L. Serrarens-Janssen, B. A. Semmekrot, V. M. J. Novotny et al., "Fetal/neonatal allo-immune thrombocytopenia (FNAIT): past, present, and future," Obstetrical and Gynecological Survey, vol. 63, no. 4, pp. 239-252, 2008.

[16] F. Boehlen, J.-F. Balavoine, and P. De Moerloose, "Severe thrombocytopenic purpura due to rubella infection in a patient with systemic lupus erythematosus," Lupus, vol. 12, no. 2, pp. 144-146, 2003.

[17] O. Olerup and H. Zetterquist, "HLA-DR typing by PCR amplification with sequence-specific primers (PCR-SSP) in 2 hours: an alternative to serological DR typing in clinical practice including donor-recipient matching in cadaveric transplantation," Tissue Antigens, vol. 39, no. 5, pp. 225-235, 1992.

[18] J.-Y. Lyou, Y.-J. Chen, H.-Y. Hu, J.-S. Lin, and C.-H. Tzeng, "PCR with sequence-specific primer-based simultaneous genotyping of human platelet antigen-1 to -13w," Transfusion, vol. 42, no. 8 , pp. 1089-1095, 2002.

[19] C.-C. Chu, H.-L. Lee, T. W. Chu, and M. Lin, "The use of genotyping to predict the phenotypes of human platelet 
antigens 1 through 5 and of neutrophil antigens in Taiwan," Transfusion, vol. 41, no. 12, pp. 1553-1558, 2001.

[20] C. Ghevaert, K. Campbell, J. Walton et al., "Management and outcome of 200 cases of fetomaternal alloimmune thrombocytopenia," Transfusion, vol. 47, no. 5, pp. 901-910, 2007.

[21] J. O. Bordin, J. G. Kelton, M. N. Warner et al., "Maternal immunization to Gov system alloantigens on human platelets," Transfusion, vol. 37, no. 8, pp. 823-828, 1997.

[22] J. E. Berry, C. M. Murphy, G. A. Smith et al., "Detection of Gov system antibodies by MAIPA reveals an immunogenicity similar to the HPA-5 alloantigens," British Journal of Haematology, vol. 110, no. 3, pp. 735-742, 2000.

[23] X. Xu, Y. Liu, Y. Ying et al., "Human platelet antigen allele frequencies and new mutations on platelet glycoprotein genes in the Chinese Han population," Transfusion Medicine, vol. 21, no. 5, pp. 330-337, 2011.

[24] S. Tanaka, S. Ohnoki, H. Shibata, Y. Okubo, H. Yamaguchi, and Y. Shibata, "Gene frequencies of human platelet antigens on glycoprotein IIIa in Japanese," Transfusion, vol. 36, no. 9, pp. 813-817, 1996.

[25] M. Landau and N. Rosenberg, "Molecular insight into human platelet antigens: structural and evolutionary conservation analyses offer new perspective to immunogenic disorders," Transfusion, vol. 51, no. 3, pp. 558-569, 2011. 


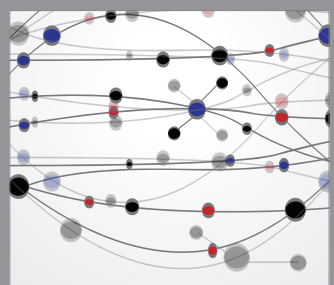

The Scientific World Journal
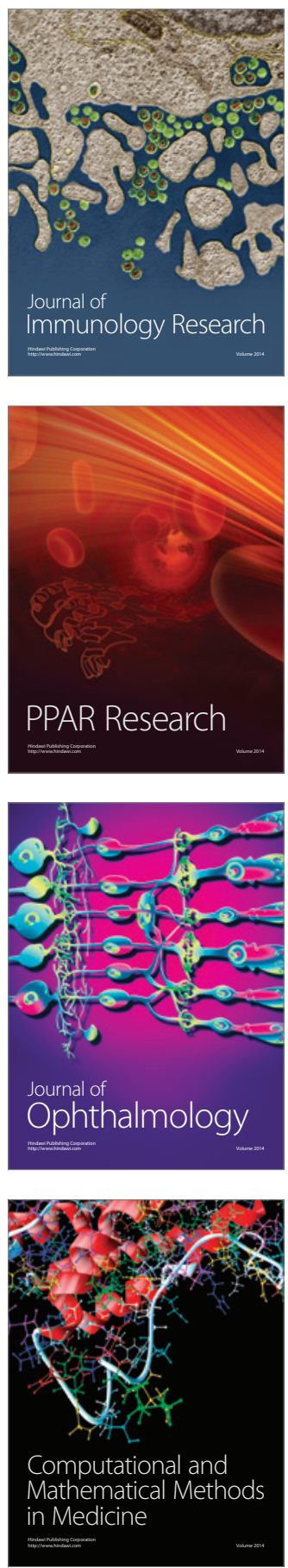

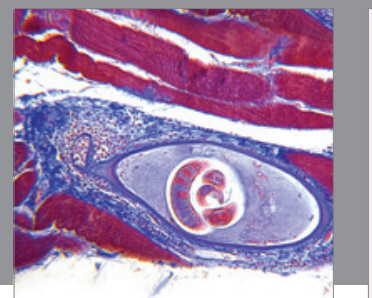

Gastroenterology

Research and Practice
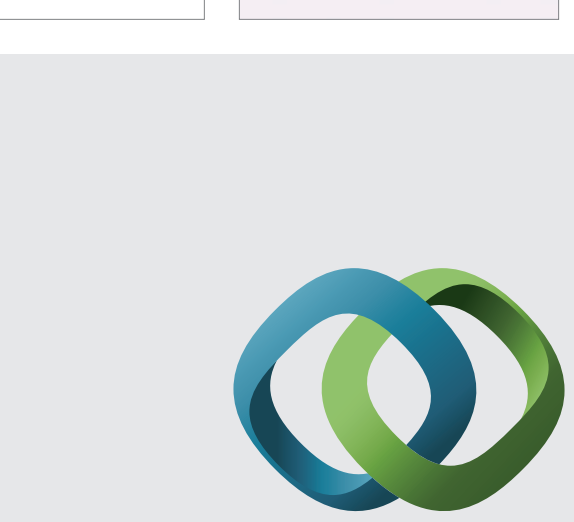

\section{Hindawi}

Submit your manuscripts at

http://www.hindawi.com
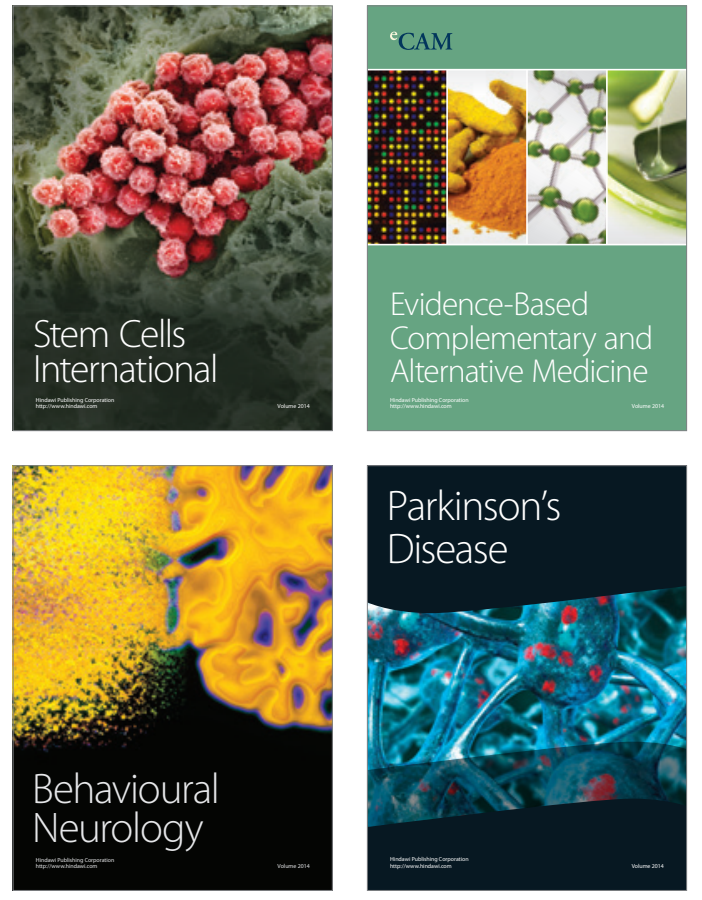
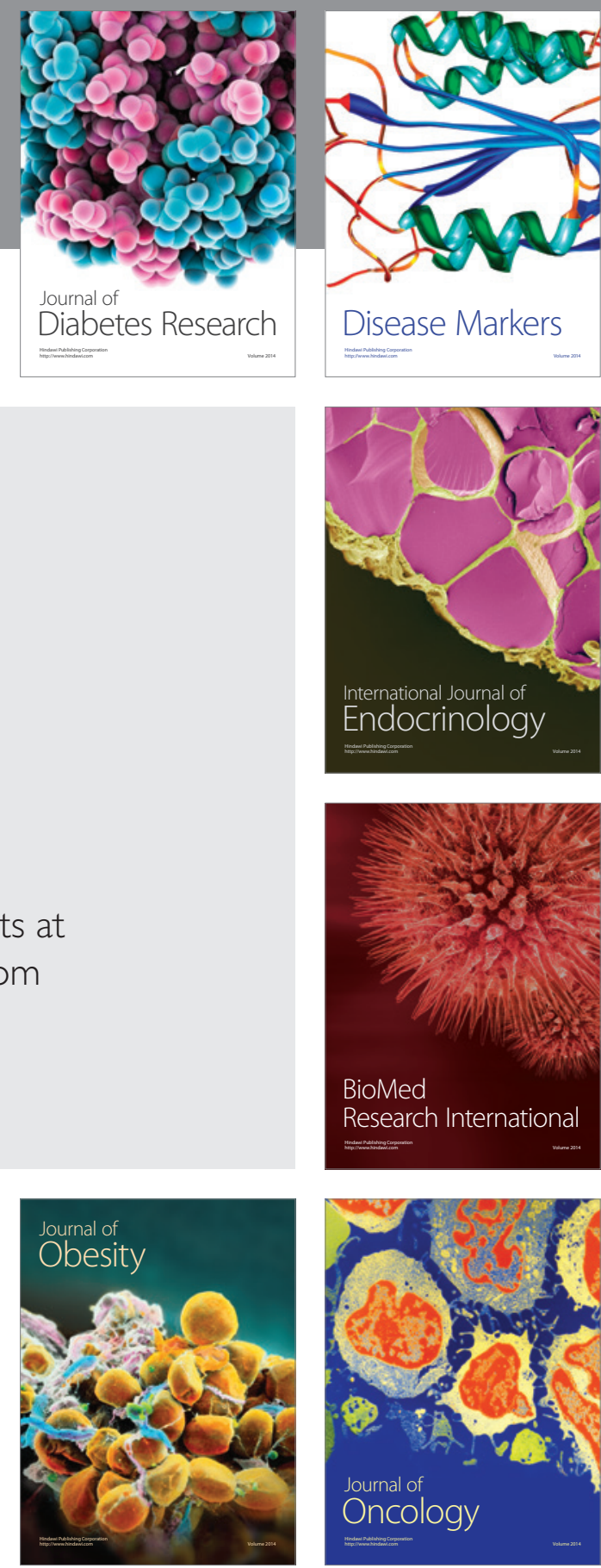

Disease Markers
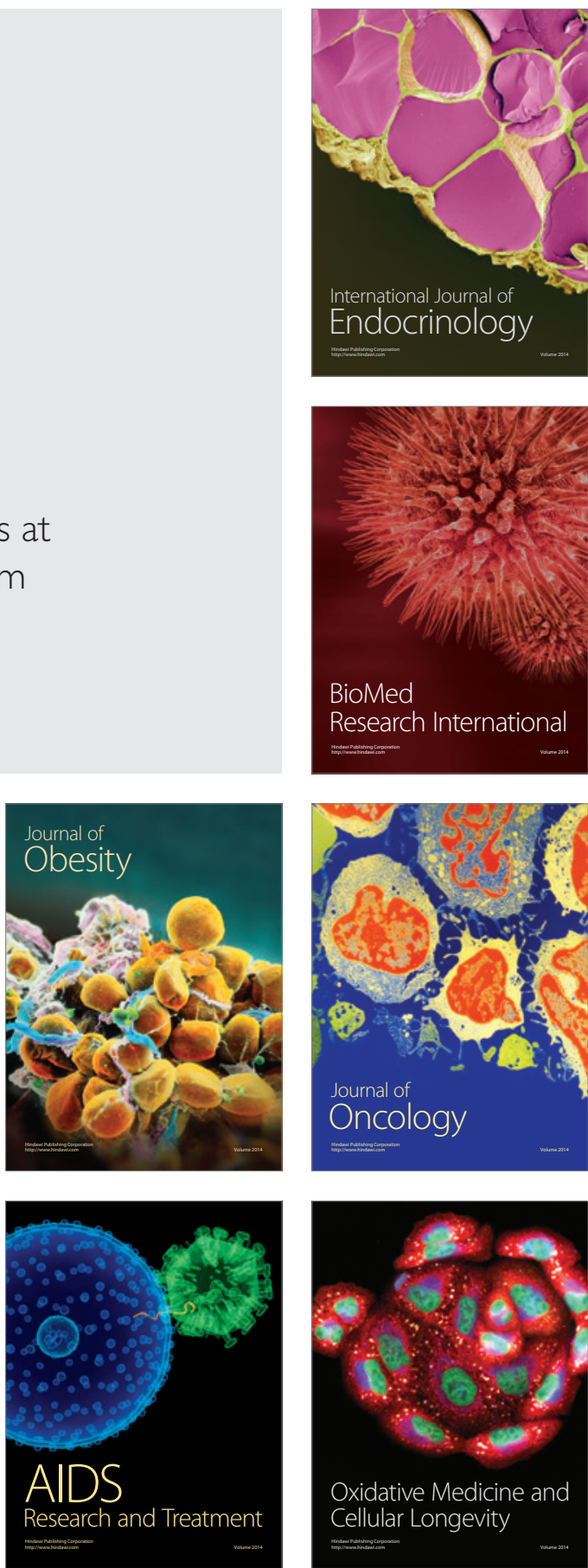